\title{
Efficacy and Safety of Teprotumumab in Thyroid Eye Disease
}

\author{
Honeylen Maryl Teo (D) \\ Terry J Smith ${ }^{2,3}$ \\ Shannon S Joseph ${ }^{2}$ \\ 'Eye Institute, St. Luke's Medical Center, \\ Metro Manila, Philippines; ${ }^{2}$ Department of \\ Ophthalmology and Visual Sciences, W.K. \\ Kellogg Eye Center, University of Michigan \\ Medical School, Ann Arbor, MI, USA; \\ ${ }^{3}$ Division of Metabolism, Endocrinology \\ and Diabetes, Department of Internal \\ Medicine, University of Michigan Medical \\ School, Ann Arbor, MI, USA
}

Correspondence: Shannon S Joseph 1000 Wall Street, Ann Arbor, MI, 48I05, USA

Tel + I-734-936-9503

Email sjshan@med.umich.edu

\begin{abstract}
Thyroid eye disease (TED; also known as thyroid-associated ophthalmopathy) is an autoimmune condition with disabling and disfiguring consequences. Teprotumumab is the first and only medication approved by the United States Food and Drug Administration for the treatment of TED. We review the efficacy and safety of teprotumumab in TED, highlighting results from the 2 randomized, double-masked, placebo-controlled trials. Postapproval case reports of teprotumumab use in patients with compressive optic neuropathy $(\mathrm{CON})$ and inactive TED were similarly favorable to those from the trials. The preliminarily results of teprotumumab for CON and inactive TED should be investigated in formal clinical trials. Teprotumumab should be avoided in pregnancy. Evidence also suggests that teprotumumab may exacerbate pre-existing inflammatory bowel disease, worsen hyperglycemia, and be associated with hearing impairment. Patients at risk for these adverse events need to be closely monitored with baseline and periodic assessments.
\end{abstract}

Keywords: monoclonal antibody, anti-IGF-IR, thyroid-associated ophthalmopathy, Graves' ophthalmopathy, autoimmune

\section{Introduction}

Thyroid eye disease (TED, also known as thyroid-associated ophthalmopathy) is a disabling and disfiguring autoimmune condition. ${ }^{1}$ The pathophysiology of TED involves a complex interplay between autoantigens including the thyroid stimulating hormone receptor (TSHR) and the insulin-like growth factor-I receptor (IGF-IR), generation of autoantibodies to these receptors, $\mathrm{T}$ and $\mathrm{B}$ lymphocytes, and various cytokines. These interactions lead to the activation of orbital fibroblasts which are responsible for the characteristic soft tissue changes of TED. ${ }^{1,2}$ Specifically, the discovery that IGF-IR forms a physical and functional complex with TSHR and that inhibition of IGF-IR results in attenuation of downstream signaling initiated by either receptor supports a central role of IGF-IR in TED pathogenesis. ${ }^{3}$

Most patients with TED have underlying Graves' disease (GD). The pathophysiology of GD also remains to be fully understood. TSHR autoantibodies play a central role in GD pathogenesis, and the titer of these autoantibodies positively correlate with TED activity and severity. ${ }^{4-8}$ Further, autoantibodies generated in patients with GD also bind to IGF-IR and can initiate signaling through the TSHR/ IGF-IR complex. ${ }^{9}$ Similar to that seen in TED pathogenesis, TSH and IGF-I pathways interact in thyroid epithelial cells and both are important in regulating thyroid hormone synthesis and participate in GD pathogenesis. ${ }^{9}$ The attainment and maintenance of the euthyroid state is associated with improvement of TED. Current 
treatment options for hyperthyroid GD include radioactive iodine (RAI) thyroid ablation, antithyroid drug (ATD), and surgical thyroidectomy, though RAI may potentially cause the development or worsening of TED. ${ }^{10}$

Based on accumulating evidence supporting the critical role of IGF-IR in TED pathogenesis, teprotumumab was developed as a potential therapy. Teprotumumab is a fully human monoclonal IgG1 antibody against IGF-IR, ${ }^{11}$ and is the only medication thus far approved by the United States (US) Food and Drug Administration (FDA) for TED. ${ }^{12}$ In this article, we review the efficacy and safety of teprotumumab in the treatment of TED, highlighting results from 2 randomized, placebo-controlled, multicenter clinical trials as well as case reports from its post-approval use. All reports were identified through literature searches using the PubMed database.

\section{Efficacy of Teprotumumab in TED Teprotumumab for Active, Moderate to Severe TED: Results of the Clinical Trials}

Two multicenter, double-masked, placebo-controlled trials were performed evaluating the efficacy and safety of teprotumumab in TED (Phase 2 NCT01868997; Phase 3 NCT03298867). Both trials enrolled patients with active, moderate to severe TED. Inclusion criteria included TED of $\leq 9$ months duration at screening, CAS of $\geq 4$ points on a 7-point scale in the more severely affected (study) eye, and no history of surgical or medical treatment for TED. History of oral glucocorticoid with a cumulative dose of $\leq 1 \mathrm{~g}$ of methylprednisolone or equivalent was allowed, after a 6-week washout period. The phase 3 trial also excluded patients with prior treatment with teprotumumab or selenium supplementation within 3 weeks of the screening period. The treatment regimen involved 8 infusions of either placebo or teprotumumab starting with the initial dose of $10 \mathrm{mg} / \mathrm{kg}$ body weight administered at week 0 , then increased to $20 \mathrm{mg} / \mathrm{kg}$, which was given every 3 weeks from weeks 3 to 21 . The endpoints were assessed at week 24 ( 3 weeks after the final infusion). ${ }^{13,14}$

The outcome measures used in these clinical trials included proptosis reduction, improved soft tissue inflammation as measured by clinical activity score (CAS), diplopia improvement, and quality of life (QOL) as measured by the Graves' ophthalmopathy-specific quality of life (GO-QOL) score. ${ }^{13,14}$ Intention-to-treat analysis was performed in both trials. A subsequent study by Kahaly et al reported the results of subgroup analyses using pooled data from both trials and off-treatment followup. ${ }^{15}$ We review the findings of both trials and the subsequent analysis below.

Both clinical trials demonstrated significant proptosis reduction in response to teprotumumab, regardless of sex and smoking status. Proptosis response was defined as reduction of $\geq 2 \mathrm{~mm}$ in the study (more severely affected) eye, without similar worsening in the fellow eye. At week 24 , there were significantly more patients who experienced proptosis response with teprotumumab compared to placebo in both clinical trials and pooled data (phase 2: 69\% or $29 / 42$ versus $20 \%$ or $9 / 45, \mathrm{p}<0.001$; phase $3: 83 \%$ or $34 / 41$ versus $10 \%$ or $4 / 42, \mathrm{p}<0.001$; pooled data: $77 \%$ or $65 / 84$ versus $15 \%$ or $13 / 87$; $<0.0001) .{ }^{13-15}$

The mean proptosis reduction from baseline was also greater with teprotumumab than placebo in both clinical trials (phase $2:-2.46 \pm 0.20 \mathrm{~mm}$ versus $-0.15 \pm 0.19 \mathrm{~mm}$; phase $3:-2.82 \pm 0.19 \mathrm{~mm}$ versus $-0.54 \pm 0.19 \mathrm{~mm}$; both with $\mathrm{p}<0.001){ }^{13,14}$ In phase 2 , more patients had $\geq 4 \mathrm{~mm}$ and $\geq 3 \mathrm{~mm}$ proptosis reductions from baseline with teprotumumab compared to placebo $(\mathrm{p}<0.001){ }^{13}$ These levels of mean proptosis reduction are similar to results attained with singlewall orbital decompression surgery. ${ }^{16,17}$ In addition, the inter-group difference in the proptosis reduction increased at subsequent time points $(p<0.001)$. Patients with the most severe proptosis at baseline were found to manifest the most significant reductions with teprotumumab therapy. The onset of therapeutic proptosis reduction was rapid. In the phase 2 trial, at week 6 , significant proptosis reduction was evident with teprotumumab compared to placebo $(43 \%$ or $18 / 42$ versus $4 \%$ or $2 / 45 ; \mathrm{p}<0.001)$. The median time to response with teprotumumab was 6.4 weeks. $^{13,14}$

Both clinical trials also demonstrated that teprotumumab significantly reduced inflammatory signs. CAS reduction was significantly greater for teprotumumab than placebo at all trial visits $(\mathrm{p}<0.001$ for all comparisons) and at week 24 (phase 2: -3.43 versus -1.85 points; phase 3: -3.7 versus -2.0 points; both $\mathrm{p}<0.001)$. CAS reduction of $\geq 3$ points was more frequent with teprotumumab versus placebo at every level of response $(\mathrm{p}<0.001)$. At week 24, more teprotumumab-treated patients had CAS of 0 or 1 compared with placebo, indicating minimal TED activity (phase 2: $69 \%$ versus $21 \%$; phase $3: 59 \%$ versus $21 \%$; both $\mathrm{p}<0.001) .{ }^{13,14}$

Overall response was defined as $\geq 2 \mathrm{~mm}$ proptosis reduction and $\geq 2$ points CAS reduction from baseline in the study eye, without a similar increase in both parameters in the non-study (fellow) eye at week 24. Overall 
response was the primary outcome in the phase 2 trial and a secondary outcome in the phase 3 trial. In both trials, more patients experienced an overall response with teprotumumab compared to placebo (phase 2: $69 \%$ or $29 / 42$ versus $20 \%$ or $9 / 45$; phase $3: 78 \%$ or $32 / 41$ versus $7 \%$ or 3/42; both $\mathrm{p}<0.001){ }^{13,14}$

Teprotumumab resulted in greater reduction in the Gorman diplopia score compared with placebo. In the phase 2 trial, more patients experienced reduction in diplopia from baseline with teprotumumab (68\% or $26 / 38$ versus $26 \%$ or $10 / 39 ; \mathrm{p}<0.001)$. The response with teprotumumab was noted across all trial visits and occurred regardless of baseline diplopia severity. ${ }^{13,14}$ In phase 3, more teprotumumab-treated patients experienced a diplopia response, defined as $\geq 1$ reduction on the Gorman diplopia scale from baseline $(68 \%$ or $19 / 28$ versus $29 \%$ or $8 / 28 ; p<0.001)$. In line with these clinical findings, orbital imaging obtained at week 24 of teprotumumab treatment in 6 patients in the phase 3 trial showed significant reduction (mean 35\%) in extraocular muscle volume. ${ }^{14}$ These findings are consistent with the known role of the IGF-IR signaling pathway in activating fibrocytes and orbital fibroblasts leading to tissue volume expansion. ${ }^{1,2,18}$

Lastly, both clinical trials demonstrated a positive impact of teprotumumab on QOL of TED patients, which was measured using GO-QOL, the 16-item (8 items each for the 2 subscales of visual function and psychosocial function from altered physical appearance) validated questionnaire. A clinically meaningful change in GO-QOL is a difference of $\geq 6$ points in one or both subscales. ${ }^{19}$ Pooled data from both trials showed that teprotumumab significantly improved the GO-QoL subscale of visual functioning (least squares [LS] mean 16.8 with standard error [SE] of 2.0 versus 6.1 with SE of 2.0; 10.7 difference, $95 \% \mathrm{CI}$ of $5.4-16.0 ; \mathrm{p}<0.001)$ and physical appearance (LE mean 13.5 SE 1.9 versus 5.8 SE 1.8, 7.7 difference, $95 \% \mathrm{CI}$ of $2.9-12.6, \mathrm{p}<0.002) .{ }^{20}$ In both trials, the GO-QOL overall score improved significantly with teprotumumab (phase 2: $17.7 \pm 2.4$ versus $6.8 \pm 2.3$ points, $\mathrm{p}<0.01$; phase 3 : $13.79 \pm 2.07$ versus $4.43 \pm 2.10$ points, $\mathrm{p}<0.001$; pooled for both trials LS mean 15.6 SE 1.6 versus 5.9 SE 1.5, 9.6 difference, 95\% CI of 5.5-13.7, $\mathrm{p}<0.001) .{ }^{13,14,20}$

The effects of teprotumumab on TED appeared to be durable. Of 37 patients completing the intervention period of the phase 2 trial, $49 \%$ (18/37) maintained a response at week 72 (51 weeks after the final dose). ${ }^{21}$ Of the 79 patients who completed the phase 3 trial, 13\% (5/39) of those treated with teprotumumab were non-responders and were re-treated. Twenty-three percent (9/39) of teprotumumab patients who experienced disease flare during the follow-up period were also re-treated in the OPTIC-X trial. Ninety-three percent (37/40) of placebo-treated patients subsequently received teprotumumab. ${ }^{15}$ During the treatment and follow-up periods of both trials, 11 patients in the placebo group received additional medical and surgical treatments for TED. Of these, 6 underwent orbital decompression surgeries. 8 patients treated with teprotumumab had additional treatments, 3 of whom underwent orbital decompression surgeries. ${ }^{15}$

In summary, teprotumumab is associated with a rapid and clinically significant reduction in proptosis, tissue inflammation, and diplopia; with improvement in quality of life. The improvements by teprotumumab in the study and fellow (contralateral) eye were similar. The response to teprotumumab appears to exhibit durability, though disease flare can occur. $^{13,14}$ The numbers needed to treat (number of patients who need to be treated with teprotumumab in order to attain one additional respective positive outcome) were 1.6 for proptosis response, 1.7 for overall response (treatment difference $60 \%, 95 \%$ CI 48-72), 2.5 for disease inactivation (treatment difference 40\%, 95\% CI 27-53), 2.5 for diplopia response (treatment difference 39\%, 95\% CI 23-55) (all $\mathrm{p}<0.0001) .{ }^{15}$ Although no published studies have directly compared teprotumumab with other treatment modalities, aggregate results concerning glucocorticoids, orbital radiotherapy (ORT), and other monoclonal antibodies in TED indicate their inferiority. ${ }^{22-30} \mathrm{~A}$ meta-analysis including randomized controlled trials showed that the use of intravenous glucocorticoids resulted to a mean CAS reduction of 2.5, TED inactivation in 59\%, and diplopia improvement in $36 \%$ of patients; intravenous glucocorticoids had little $(1.14 \mathrm{~mm})$ to no effect on proptosis reduction. ${ }^{24,25}$ ORT improved extraocular muscle motility (odds ratio of 4.88 , 95\% CI 1.93-12.34) but had no significant effect on proptosis reduction, CAS reduction, or the need for additional treatment in moderate to severe TED. ${ }^{26,27}$ Two randomized controlled trials showed that rituximab had no significant effect on proptosis reduction and diplopia in patients with active, moderate to severe TED. While Stan et al reported no difference between rituximab and placebo in CAS reduction at 24 and 52 weeks, Salvi et al showed the superiority of rituximab over intravenous glucocorticoids in reducing CAS at 24 weeks. The favorable finding by Salvi et al may be due to the shorter TED duration (mean of 4.5 months compared 
with mean of 11.2 months for the Stan et al trial) and the fewer patients who underwent previous radioactive iodine therapy. $^{28,29}$ The effect of tocilizumab versus placebo on CAS reduction and proptosis reduction at week 40 was not significant. Tocilizumab had no effect in patients with steroid-resistant, moderate to severe TED on diplopia or quality of life. ${ }^{30}$

\section{Teprotumumab for Compressive Optic Neuropathy in TED}

Compressive optic neuropathy (CON) can occur in TED as a consequence of enlarged extraocular muscles at the orbital apex, and less commonly from stretching of the optic nerve from severe proptosis. Patients with CON were excluded from both clinical trials. Nevertheless, since the FDA approval of teprotumumab, 6 publications have reported on 15 cases demonstrating favorable effects of teprotumumab for CON (Table 1). ${ }^{31-36}$

All 15 patients had failed to improve with or were not candidates for conventional treatments including systemic corticosteroids, orbital radiotherapy, and/or surgical decompression. All had poor optic nerve function, which was characterized by decreased visual acuity (VA) and/or color vision, presence of relative afferent pupillary defect (RAPD), and visual field (VF) defect. For this review, a patient was considered to have "resolution of CON" with treatment if they had a final VA of 20/20 or baseline VA in the involved eye, normal color vision, and resolution of abnormal optic nerve function findings including RAPD, optic nerve changes, or VF defect. A patient was considered to have "improved CON" if they had improved yet persistently abnormal optic nerve function. Of the 15 reported cases, 9 had complete resolution of CON while 6 cases had improved CON. ${ }^{31-36}$ The onset of improvement was as early as after 2 doses of teprotumumab in 6 of these cases. ${ }^{32,36}$

Data on proptosis reduction and CAS improvement were also available in 13 of the 15 reported cases. Twelve of 13 cases had $\geq 2 \mathrm{~mm}$ proptosis reduction in the involved eye (range, $2 \mathrm{~mm}$ to $10 \mathrm{~mm}$ ). All 13 cases had a final CAS of 0 to 1 (CAS reduction range, 4 to 7 ). As observed in the clinical trials, the onset of response was rapid. After only 2 doses, proptosis reduction was noted in 5 of 13 cases and CAS improvement in 9 of 13 cases. ${ }^{31-34,36}$ In addition, these responses persisted at 3-33 weeks after the final 8th dose. $^{31-33,36}$ Radiographic data was available for 7 cases, and all showed reduction in both extraocular muscle size and apical crowding, supporting the clinical evidence of
CON resolution or improvement. While these reports suggest that teprotumumab may be an effective therapy for CON, further studies should help identify clinical factors that would be predictive of a positive response to teprotumumab, including duration and/or severity of CON. For example, teprotumumab may only have favorable results in $\mathrm{CON}$ when the optic nerve has not yet reached the irreversible atrophic state.

\section{Teprotumumab for Inactive TED}

Patients with inactive TED were also excluded from both clinical trials. However, 2 subsequent reports of 5 patients have demonstrated favorable effects of teprotumumab in chronic inactive TED (Table 2). Ugradar et al reported 4 consecutive patients with "non-inflammatory" TED, each with CAS of $\leq 1$ for at least 4 months, diplopia scores of 0 , but all had persistent bilateral proptosis. All four patients, each without previous history of orbital surgery or radiotherapy, were treated with teprotumumab. After 8 infusions, all 4 patients demonstrated $\geq 2 \mathrm{~mm}$ proptosis reduction (mean reduction $2.6 \mathrm{~mm}$, standard deviation $1.2, \mathrm{p}<0.01$ ) in the more severely affected eye. The CAS and diplopia scores remained 0 throughout the treatment. It is important to note that while these four patients described by Ugradar et al were defined as clinically "non-inflammatory" based on CAS of $\leq 1$, the duration of disease were only $4,5,7$, and 12 months. Thus, it is conceivable that patients may have early, mildly active disease rather than chronic, inactive disease. This issue highlights the imprecision of judging TED activity on the basis of CAS. A separate report by Ozzello et al described the case of a 50-year-old man with asymmetric bilateral proptosis of $\geq 3$ years duration that had been stable for 2 years. Treatment with teprotumumab was associated with $5 \mathrm{~mm}$ bilateral proptosis reduction after 2 infusions. CAS improved from 1 to 0 with the resolution of orbital pain after 3 infusions. ${ }^{37,38}$

Currently, chronic, inactive TED is predominantly managed with surgical rehabilitation as needed. The reports above suggest the potential of teprotumumab as disease-modifying in chronic inactive TED. ${ }^{37,38}$ While the initial findings of Ugradar et al showed IGF-IR overexpression in orbital fibroblasts of "non-inflammatory" TED compared to controls, ${ }^{37}$ more rigorous clinical studies are required to fully elucidate the role of teprotumumab in treating inactive TED. A 24-week, randomized, placebo-controlled, multicenter trial (Phase 4 NCT04583735) to evaluate the efficacy and safety of 


\begin{tabular}{|c|c|c|c|c|c|c|c|c|c|c|c|c|c|c|c|c|c|}
\hline $\begin{array}{l}\stackrel{n}{0} \\
\dot{\nu} \\
\tilde{g}\end{array}$ & 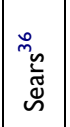 & $\Sigma$ & $\stackrel{2}{2}$ & $z$ & ని & $\begin{array}{l}\text { 今े } \\
\tilde{\omega}\end{array}$ & ว & & 옹 & 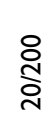 & $\underline{\underline{\Sigma}}$ & $\underline{\underline{\Sigma}}$ & $\stackrel{m}{m}$ & 0 & כ & in & $\begin{array}{l}\overline{0} \\
\stackrel{y}{\bar{y}} \\
\bar{y} \\
\bar{\alpha}\end{array}$ \\
\hline $\begin{array}{l}\square \\
\\
\tilde{J}\end{array}$ & 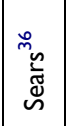 & $\Sigma$ & 우 & $z$ & 으 & $\approx$ & กั & & ָั & ָั & $\succ$ & $\succ$ & $\begin{array}{l}\sigma \\
\kappa\end{array}$ & $n$ & $\succ$ & $\stackrel{m}{m}$ & ż \\
\hline $\begin{array}{l}\frac{m}{y} \\
\stackrel{y}{J} \\
\text { J }\end{array}$ & 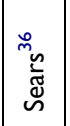 & $\Sigma$ & in & $z$ & తి & 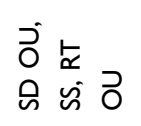 & ว & & $\stackrel{n}{\stackrel{2}{\sim}}$ & ঙ্ণি & $\succ$ & $\succ$ & $=$ & $\sigma$ & כ & $\approx$ & 产 \\
\hline 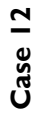 & 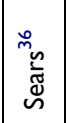 & ч & $\stackrel{M}{\wedge}$ & $z$ & $N$ & $\approx$ & Оิ & & ্ָ & ָั & $\succ$ & $\overleftarrow{z}$ & $\hat{n}$ & $\wedge$ & $\supset$ & 0 & \\
\hline $\begin{array}{l}\bar{\Sigma} \\
\bar{y} \\
\tilde{J}\end{array}$ & 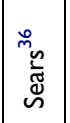 & ч & 缉 & $z$ & $\sim$ & $\begin{array}{l}\text { के } \\
\text { जे o }\end{array}$ & రొ & & ָั & $\stackrel{\sim}{\stackrel{2}{*}}$ & $\succ$ & $\succ$ & $\begin{array}{l}\stackrel{n}{+} \\
\stackrel{n}{m} \\
\dot{m}\end{array}$ & $\sigma$ & $\succ$ & 0 & \\
\hline 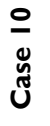 & 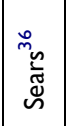 & ч & $\stackrel{m}{\wedge}$ & $\frac{\bar{o}}{2}$ & $\simeq$ & $\begin{array}{l}0 \\
\tilde{n} \\
\tilde{n}\end{array}$ & Oొ & & ্ָ & ָั & 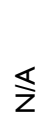 & $\succ$ & $\frac{0}{\sigma^{\circ}}$ & in & כ & $\underline{0}$ & \\
\hline $\begin{array}{l}0 \\
\text { ỹ } \\
\text { Ũ }\end{array}$ & 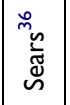 & ч & $\widetilde{\sigma}$ & $z$ & 0 & $\varkappa$ & О & & 号 & 旁 & $\overleftarrow{z}$ & $\succ$ & $\begin{array}{l}\text { in } \\
\dot{\sigma}^{2}\end{array}$ & $\sigma$ & $>$ & $\pi$ & 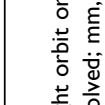 \\
\hline $\begin{array}{l}\infty \\
0 \\
\tilde{y} \\
\text { U }\end{array}$ & 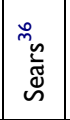 & 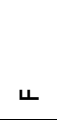 & ‡ & $z$ & $\frac{2}{2}$ & $\approx$ & $\widehat{\partial}$ & & 怘 & $\begin{array}{l}\stackrel{8}{0} \\
\stackrel{\text { İ }}{\text { iे }}\end{array}$ & 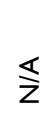 & 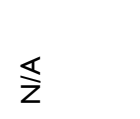 & $\begin{array}{l}m \\
m\end{array}$ & $\sigma$ & $\succ$ & $m$ & \\
\hline ĩ & 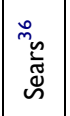 & 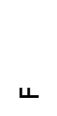 & 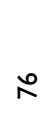 & $z$ & $\frac{\text { ని }}{\wedge}$ & $\begin{array}{l}0 \\
\tilde{\omega} \\
\tilde{\omega}\end{array}$ & Ј & & 怘 & $\begin{array}{l}\stackrel{8}{\circ} \\
\stackrel{\infty}{\text { İ }}\end{array}$ & 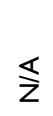 & $\overleftarrow{z}$ & $\stackrel{N}{m}$ & 0 & $\supset$ & $\underline{0}$ & \\
\hline $\begin{array}{l}0 \\
\vdots \\
\tilde{y} \\
\tilde{J}\end{array}$ & 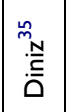 & 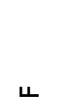 & 2 & D & N & $\approx$ & Оิ & & D & 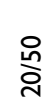 & $\succ$ & $\succ$ & כ & $\supset$ & כ & $\overleftarrow{z}$ & \\
\hline $\begin{array}{l}n \\
\tilde{y} \\
\tilde{y} \\
0\end{array}$ & 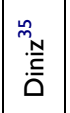 & 4 & $\infty_{\infty}$ & כ & $\sigma$ & 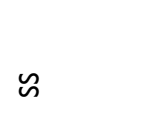 & రิ & & D & ڤ̊ & $\succ$ & $\underline{\underline{\Sigma}}$ & כ & $\supset$ & כ & $\stackrel{\S}{z}$ & \\
\hline $\begin{array}{l}+ \\
\dot{u} \\
\tilde{y} \\
0\end{array}$ & 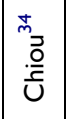 & 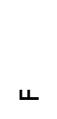 & $\stackrel{\infty}{\circ}$ & $z$ & $m$ & $\tilde{\omega}$ & రิ & & $\stackrel{\sim}{\check{N}}$ & $\stackrel{\tilde{N}}{\stackrel{\sim}{\sim}}$ & 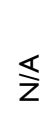 & 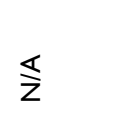 & $\begin{array}{c}m \\
\stackrel{m}{+}\end{array}$ & 0 & כ & 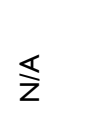 & \\
\hline $\begin{array}{l}m \\
\text { J } \\
\text { Ũ }\end{array}$ & 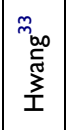 & 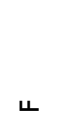 & $\bar{\infty}$ & כ & $\infty$ & $\approx$ & $\widehat{0}$ & & 产 & ஓ্̀ & $\succ$ & 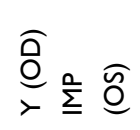 & $\begin{array}{l}\stackrel{20}{+} \\
\text { in }\end{array}$ & 0 & $\succ$ & 0 & $\dot{\varepsilon}$ \\
\hline $\begin{array}{l}\text { N } \\
\tilde{y} \\
\tilde{J}\end{array}$ & 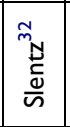 & $\Sigma$ & $\widetilde{\sigma}$ & $\succ$ & 0 & 巳̊ & ชิ & & ָั & 옹 & $\hat{\mathbf{z}}$ & 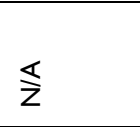 & $\begin{array}{l}\text { in } \\
\text { í }\end{array}$ & in & $\succ$ & $\sigma$ & \\
\hline $\begin{array}{l}\bar{\Phi} \\
\tilde{J} \\
\end{array}$ & 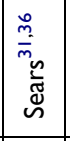 & $\Sigma$ & $\stackrel{\text { 乌 }}{2}$ & \begin{tabular}{|l}
$\frac{\vdots}{2}$ \\
$\vdots$ \\
\end{tabular} & $\stackrel{\infty}{\underline{\infty}}$ & $\begin{array}{l}\text { s } \\
\text { जे }\end{array}$ & $\widehat{0}$ & & i্ & ్ㅗ & $\succ$ & $\succ$ & $\hat{n}$ & $\nabla$ & $\succ$ & 요 & \\
\hline & 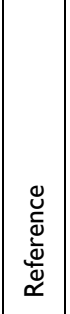 & હ & 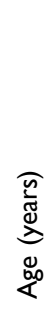 & \begin{tabular}{|l|}
$\overline{\bar{\nu}}$ \\
$\overline{0}$ \\
$\underline{\tilde{n}}$
\end{tabular} & 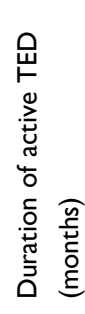 & 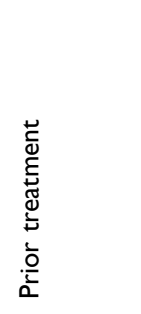 & 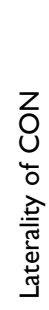 & 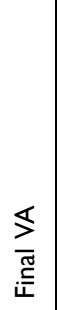 & Оิ & oิ & 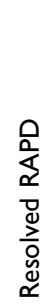 & 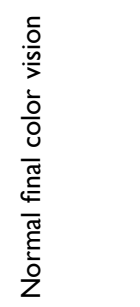 & 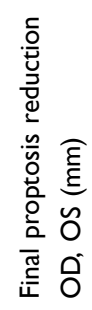 & 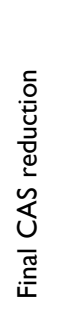 & 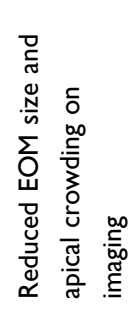 & 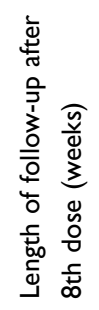 & 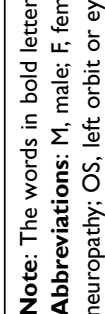 \\
\hline
\end{tabular}


Table 2 Clinical and Demographic Details of "Non-Inflammatory” or Inactive TED Patients Treated with Teprotumumab

\begin{tabular}{|c|c|c|c|c|c|}
\hline & Case I & Case 2 & Case 3 & Case 4 & Case 5 \\
\hline Reference & Ugradar $^{37}$ & Ugradar $^{37}$ & Ugradar $^{37}$ & Ugradar $^{37}$ & Ozzello ${ }^{38}$ \\
\hline Sex & $\mathrm{F}$ & $\mathrm{F}$ & $\mathrm{F}$ & M & $\mathrm{F}$ \\
\hline Age (years) & 22 & 32 & 47 & 21 & 50 \\
\hline Smoker & $\mathrm{N}$ & $\mathrm{N}$ & $\mathrm{N}$ & $\mathrm{N}$ & $Y$ \\
\hline Duration of TED (months) & 12 & 7 & 4 & 5 & 24 \\
\hline Prior treatment & SS & SS & SS & SS & None \\
\hline Final proptosis reduction after teprotumumab treatment $\mathrm{OD}, \mathrm{OS}(\mathrm{mm})$ & 3,4 & I, 2 & 3,2 & 4,3 & $6,6^{\mathrm{a}}$ \\
\hline CAS at baseline & 0 & 0 & 0 & 0 & 1 \\
\hline Reduced EOM size on orbital imaging & $\mathrm{N} / \mathrm{A}$ & N/A & N/A & N/A & Y \\
\hline
\end{tabular}

Note: The words in bold letters are headers of each column.

Abbreviations: F, female; M, male; N, no; Y, yes; TED, thyroid eye disease; SS, systemic steroids; mm, millimeter; OD, right orbit; OS, left orbit; CAS, clinical activity score; EOM, extraocular muscle; N/A, not applicable; a, after only 3 doses; no details on subsequent doses.

teprotumumab in treating patients with chronic inactive TED is currently in the recruitment process. ${ }^{39}$

\section{Safety of Teprotumumab as a Therapy for TED}

Teprotumumab is a fully human IgG1 monoclonal inhibitory antibody against IGF-IR. Since it was initially developed for cancer therapy, studies on the tolerability and adverse effects were originally performed in patients with solid tumors including sarcomas. Early phase clinical trials revealed that the drug was well-tolerated. ${ }^{40-43}$ There was no dose-limiting toxicity associated with weekly infusions of $9 \mathrm{mg} / \mathrm{kg}$; the maximum tolerated dose was not reached. ${ }^{40}$ Safety results from the clinical trials for TED and post-approval reports are discussed below (Table 3).

\section{Safety of Teprotumumab for Active, Moderate to Severe TED: Results of the Clinical Trials}

Adverse events (AE) of any cause were defined as those that occurred between the administration of the first dose and 30 days after the administration of the final dose (prior to week 25). Some patients had multiple AEs. In both trials, the most common AEs reported with teprotumumab included muscle spasms ( $25 \%$ or $21 / 84)$, nausea $(17 \%$ or $14 / 84)$, alopecia $(13 \%$ or $11 / 84)$, diarrhea (12\% or $10 / 84)$, fatigue $(12 \%)$, hearing impairment $(10 \%$ or $8 / 84)$, and hyperglycemia ( $8 \%$ or $7 / 84)$. AEs of special interest, which are AEs that potentially have mechanistic ties to teprotumumab, included infusionrelated reactions, muscle spasms, diarrhea, hearing impairment, and hyperglycemia. ${ }^{13,14}$ In the pooled follow-up study of both trials at weeks 28 and 72, AEs of special interest in the teprotumumab group included 1 case of muscle spasm, 2 cases of hearing impairment, and 2 cases of hyperglycemia. ${ }^{15}$

The severity of AEs in both trials was graded based on version 4.03 of the National Cancer Institute Common Terminology Criteria for AEs. ${ }^{13,14}$ Grade 1 or mild AEs are associated with asymptomatic or mild symptoms; intervention is not indicated. Grade 2 or moderate AEs limit age-appropriate instrumental activities of daily living; they require minimal, local, or non-invasive intervention. ${ }^{44}$ Most AEs were mild to moderate in severity for both teprotumumab and placebo groups (94\% or 63/ 67 versus $98 \%$ or $59 / 60$ ). Most AEs resolved while the patients continued in their study group. ${ }^{13,14}$ The pooled week 28 and 72 follow-up analysis of both trials showed that AEs in $90 \%(26 / 29)$ of patients who received teprotumumab and $100 \%(9 / 9)$ patients who received placebo were mild to moderate. ${ }^{15}$

Serious AEs were uncommon in both trials. Of the serious AEs occurring in the teprotumumab group (8\% or $7 / 84)$ in both trials, $4 \%(3 / 84)$ were considered by the trial investigators as possibly related to teprotumumab and led to study intervention discontinuation. These included infusion reaction, diarrhea, and transient (less than 24hour) cognitive changes with a provisional diagnosis of Hashimoto's encephalopathy. ${ }^{13,14}$

\section{Teprotumumab and Infusion-Related Reaction}

Intravenous administration of monoclonal antibodies can result in infusion-related reactions. These reactions can be anaphylactic or anaphylactoid from cytokine release. Two patients in the phase 3 trial developed infusion reaction to 
Table 3 Clinical and Demographic Details of Patients Developing Adverse Events with Teprotumumab for TED

\begin{tabular}{|c|c|c|c|c|c|c|c|c|c|}
\hline & Case I & Case 2 & Cases 3-5 & Case 6 & Case 7 & Case 8 & Case 9 & Case 10 & Case II \\
\hline Reference & Ashraf ${ }^{48}$ & $\mathrm{Safo}^{49}$ & Kossler ${ }^{52}$ & Kossler ${ }^{52}$ & Belinsky ${ }^{53}$ & Belinsky $^{53}$ & Belinsky $^{53}$ & Belinsky $^{53}$ & Hoang ${ }^{63}$ \\
\hline Sex & $\mathrm{F}$ & $\mathrm{F}$ & $\mathrm{N} / \mathrm{A}$ & N/A & $\mathrm{F}$ & $\mathrm{F}$ & $\mathrm{F}$ & $\mathrm{F}$ & M \\
\hline Age (years) & 45 & 46 & $\mathrm{~N} / \mathrm{A}$ & $N / A$ & 77 & 68 & 34 & 48 & 76 \\
\hline Smoker & $\mathrm{N}$ & $\mathrm{N}$ & N/A & $N / A$ & Previous & $N / A$ & $N / A$ & $N / A$ & N/A \\
\hline $\begin{array}{l}\text { Duration } \\
\text { of TED (in } \\
\text { months) }\end{array}$ & 6 & 2 & N/A & $N / A$ & 6 & $N / A$ & $N / A$ & $N / A$ & N/A \\
\hline $\begin{array}{l}\text { Prior } \\
\text { treatment }\end{array}$ & None & SS & $\mathrm{N} / \mathrm{A}$ & $N / A$ & SS & SS, SD OD & $N / A$ & None & None \\
\hline $\begin{array}{l}\text { Presenting } \\
\text { feature }\end{array}$ & $\begin{array}{l}\text { Bowel } \\
\text { urgency, } \\
\text { diarrhea, } \\
\text { HC, Abd } \\
\text { pain }\end{array}$ & $\begin{array}{l}\text { Fecal } \\
\text { urgency, } \\
\text { HC }\end{array}$ & $\begin{array}{l}\text { SNHL in } 2 \\
\text { of } 3 \\
\text { patients }\end{array}$ & $N / A$ & $\mathrm{~N} / \mathrm{A}$ & $\begin{array}{l}\text { Decreased } \\
\text { hearing and } \\
\text { muffled hearing } \\
\text { (worse AS) }\end{array}$ & $\begin{array}{l}\text { Difficulty } \\
\text { hearing } \\
\text { background } \\
\text { noise AS }\end{array}$ & $\begin{array}{l}\text { Fullness } \\
\mathrm{AU}\end{array}$ & $\begin{array}{l}\text { Behavior changes, } \\
\text { Confab, memory } \\
\text { deficit, delirium, } \\
\text { delusion }\end{array}$ \\
\hline $\begin{array}{l}\text { Adverse } \\
\text { event (AE) }\end{array}$ & IBD & $\begin{array}{l}\text { Ulcerative } \\
\text { colitis } \\
\text { (IBD) }\end{array}$ & $\begin{array}{l}\text { Hearing } \\
\text { impairment }\end{array}$ & $\begin{array}{l}\text { Hearing } \\
\text { impairment }\end{array}$ & $\begin{array}{l}\text { Hearing } \\
\text { impairment }\end{array}$ & $\begin{array}{l}\text { Hearing } \\
\text { impairment }\end{array}$ & $\begin{array}{l}\text { Hearing } \\
\text { impairment }\end{array}$ & $\begin{array}{l}\text { Hearing } \\
\text { impairment }\end{array}$ & $\begin{array}{l}\text { Rapid cognitive } \\
\text { decline }^{\text {a }}\end{array}$ \\
\hline $\begin{array}{l}\text { Onset of } \\
\text { AE (after } \\
x \text { doses) }\end{array}$ & 6 & 5 & $\sim 3.6$ & $\sim 3.6$ & 3 & 3 & 8 & 4 & 4 \\
\hline $\begin{array}{l}\text { Resolution } \\
\text { of } A E\end{array}$ & $\mathrm{~N}$ & $\mathrm{~N}$ & $\mathrm{~N}$ & Imp & $\mathrm{N}$ & $\mathrm{N}$ & $Y$ & Imp & Y \\
\hline
\end{tabular}

Note: The words in bold letters are headers of each column.

Abbreviations: F, female; N/A, not applicable; M, male; TED, thyroid eye disease; SS, systemic steroids; SD, surgical decompression; OD, right orbit; HC, hematochezia; Abd, abdominal; SNHL, sensorineural hearing loss; AS, left ear; AU, both ears; Confab, confabulation; AE, adverse event; IBD, inflammatory bowel disease; $x$, a certain number; N, No; Imp, improved, not resolved; Y, yes; a, causal relationship with teprotumumab cannot be established.

teprotumumab. Neither was anaphylactic. One patient developed the reaction during the initial infusion and discontinued the trial drug despite resolution with hydrocortisone within 2 hours. The other patient was pre-medicated thereafter, received subsequent infusions at a slower rate, and completed the study. No antidrug antibodies were detected in those patients who received teprotumumab. ${ }^{13,14}$ Most infusion-related reactions occur during or within 90 minutes of the first infusion and are mild in severity. These can be managed by discontinuation of the infusion, and evaluation of vital signs and airway integrity. The need for epinephrine, anti-histamine, or corticosteroids should be determined; resuming the infusion at a slower rate and titrated to tolerance is recommended. Pre-medication with anti-histamine, antipyretic, and corticosteroid may be considered prior to subsequent infusions, which should initially be given at a slower rate. $^{45}$

\section{Teprotumumab and Muscle Spasm}

Muscle spasm was the most frequently reported $\mathrm{AE}$ for teprotumumab, occurring at a higher incidence compared to placebo $(25 \%$ or $21 / 84$ versus $7 \%$ or $6 / 86)$ in both trials. IGF-I is thought to play an important role in skeletal muscle growth, repair, and prevention of degeneration. ${ }^{46}$ Inhibition of IGF-IR may lead to muscle spasm, but the mechanism has yet to be determined. The most commonly affected body areas were the lower limbs. No clinically relevant laboratory abnormalities were identified, although serum magnesium levels were not tested. Most cases were mild in severity; moderate cases were reported for 5 patients in total in the teprotumumab group in both trials. None discontinued treatment. ${ }^{13,14,20}$ Treatment measures could include muscle relaxants and supplementation with vitamin B, calcium, and magnesium. 


\section{Teprotumumab and Inflammatory Bowel Disease}

Teprotumumab may reactivate inflammatory bowel disease (IBD) in those patients with a history of the disease. IGF-I has been shown to induce proliferation of regulatory $\mathrm{T}$ cells and halt the progression of autoimmune diseases in the gut. ${ }^{47}$ In the phase 2 trial, diarrhea was reported in $14 \%(6 / 43)$ of teprotumumab-treated patients. One of these 6 cases was classified as a serious AE. The patient had a 6-month history of ulcerative colitis who developed severe diarrhea while receiving teprotumumab. Another patient was diagnosed by colonoscopy with ileitis and colitis 3 months into the study and was treated and recovered from IBD while continuing to receive teprotumumab. These after study observations led to the exclusion of patients with pre-existing IBD in the phase 3 trial. ${ }^{13,14}$

Subsequently, Ashraf et al and Safo et al independently reported two women in their 5th decade of life who developed new-onset IBD during teprotumumab therapy for active TED. After 5-6 infusions, both developed bowel urgency and hematochezia. Colonoscopy and biopsy confirmed the diagnosis of ulcerative colitis (UC) in both. Teprotumumab was discontinued for both while the patients underwent treatment for UC. Their gastrointestinal symptoms persisted for 1.5-2 months after the seventh infusion of teprotumumab. Of note, both patients had a family history of UC; one had at least 1 episode of bilateral uveitis of unknown etiology 2 years prior to the diagnosis of UC. ${ }^{48,49}$

Teprotumumab may exacerbate pre-existing IBD. Patients should be monitored for disease flare and the drug must be discontinued if IBD worsens. In addition, identifying treatment candidates with family histories of IBD should aid in stratifying risk as up to $12 \%$ of IBD cases are familial and family history is the strongest recognizable risk factor. ${ }^{50,51}$

\section{Teprotumumab and Hearing Impairment}

Hearing impairment was one of the most common AEs associated with teprotumumab in both clinical trials. ${ }^{13,14}$ None of the placebo patients developed hearing impairment while nearly $10 \%(8 / 84)$ of the teprotumumab-treated patients did so. In the phase 2 trial, $7 \%$ (3/43) developed hearing abnormalities with teprotumumab; 1 resolved, 1 had partial improvement, and 1 required evaluation for hearing aids. One patient developed mild hyperacusis before the fifth dose; audiogram performed on week 16 revealed mild bilateral hearing loss.
The bilateral hearing impairment resolved at week 35 . Another patient with moderate eustachian tube dysfunction developed moderate unilateral deafness 16 weeks after completing teprotumumab therapy; audiogram performed on week 39 showed moderate mixed hearing loss. The eustachian tube dysfunction resolved at week 68; unilateral moderate, mixed hearing loss partially improved by week 71 . A third patient with preexisting intermittent tinnitus from loud noise exposure developed moderate high frequency sensorineural bilateral hearing loss at the time of the eighth dose. Evaluation for hearing aids was recommended. ${ }^{13,46}$ In the phase 3 trial, 12\% (5/41) developed spontaneously resolving hearing impairment with teprotumumab: 2 cases of mild to moderate hypoacusis; 1 case of moderate deafness; 1 case of mild autophony; and 1 case of mild patulous eustachian tube. ${ }^{14}$ The hearing impairments developed after 4-8 infusions. All patients in the teprotumumab group completed therapy. Of note, baseline audiograms were not part of the screening protocol in either trial. Further, not all patients who reported hearing impairment underwent formal audiology testing. ${ }^{13,14}$

Kossler et al published a retrospective series of 28 TED patients treated with teprotumumab and found that 13 patients $(46 \%)$ experienced hearing symptoms after a mean of 3.6 infusions. The most commonly reported symptoms were autophony, hearing loss, or muffled hearing. Three patients developed sensorineural hearing loss (SNHL) and did not experience significant improvement 3 months after drug discontinuation. One patient developed patulous eustachian tube (PET), with some improvement of her symptoms after drug discontinuation. ${ }^{52}$ No other details were provided for the 9 remaining patients who were reported to have hearing symptoms. Another retrospective series by Belinsky et al of 28 patients with TED treated with teprotumumab found 4 patients (14\%) to have hearing symptoms after receiving at least 3 infusions. All 4 patients developed SNHL with abnormal audiogram findings. ${ }^{53}$ One patient discontinued treatment after 7 infusions; the others completed all 8 infusions. In 3 patients, the hearing impairment persisted 2,3, and 7 months after the final dose. One patient who developed hearing symptoms after 4 doses gradually improved and completed 8 doses. ${ }^{53}$

The frequency, magnitude, reversibility, and mechanism of the hearing impairment associated with teprotumumab remains unclear. Studies have shown the crucial role of IGF-I in the development, survival, and maintenance of cochlear hair cells, regeneration of cochlear synapses, and synaptic neurotransmission in the cochlear nuclei. ${ }^{54-56}$ IGF-I appears to protect cochlear hair cells 
from trauma due to loud noise, medication toxicity, and ischemia. $^{54-56}$ It is possible that IGF-IR inhibition by teprotumumab may alter receptor signaling and result in SNHL. Eustachian tube dysfunction and autophony, which were identified in both clinical trials and one case series, may be due to atrophy of the nasopharyngeal fat pad, resulting in Eustachian tube dysregulation. ${ }^{13,14,53}$ Further studies are needed to better understand the association between hearing impairment and teprotumumab therapy. It may be prudent to obtain audiologic testing at baseline, during, and after completion of teprotumumab therapy. Patients should also be closely monitored for hearing symptoms through the course of treatment. ${ }^{52,53}$

\section{Teprotumumab and Hyperglycemia}

Hyperglycemia is an AE of special interest associated with teprotumumab therapy, ${ }^{13,14,39-41}$ and was more commonly reported in patients with pre-existing diabetes mellitus or impaired glucose tolerance. ${ }^{13}$ This finding is likely related to reduced insulin sensitivity from IGF-IR inhibition, a coreceptor of the insulin receptor. ${ }^{13,57}$

In the phase 2 trial, hyperglycemia occurred in $12 \%(5 / 43)$ of teprotumumab-treated patients. ${ }^{13}$ Hyperglycemia was consistently grade 1 (asymptomatic or mild) among non-diabetics, and grade 2 or 3 (severe or medically significant but not immediately life-threatening) among patients with baseline diabetes. ${ }^{43}$ In the phase 3 trial, $5 \%$ (2/41) of patients who received teprotumumab developed grade 1 (mild) hyperglycemia. In both trials, hyperglycemia occurred in more patients who received teprotumumab compared to placebo $(10 \%$ or $8 /$ 84 versus $1 \%$ or $1 / 86$ ). Five out of 8 patients who developed hyperglycemia in the teprotumumab group had pre-existing diabetes mellitus or impaired glucose tolerance at baseline. In all cases, hyperglycemia was managed through diabetes medication adjustment. All events resolved either during or immediately following treatment; none led to teprotumumab discontinuation. $^{13,14}$

In summary, hyperglycemia can occur during teprotumumab therapy, especially in patients with pre-existing impaired glucose tolerance. Prior to initiating treatment with teprotumumab, baseline glucose and HbA1c testing and optimizing glycemic control are strongly recommended. During teprotumumab treatment, patients with history of diabetes or pre-diabetes should be closely monitored for the development of hyperglycemia and treated accordingly in collaboration with endocrinologists or primary care providers. ${ }^{13,14,58}$

\section{Teprotumumab in the Pregnant and Pediatric Populations}

IGF-I is an important mediator of normal and abnormal growth, and the IGF-I pathway plays a critical role in both intrauterine and postnatal development or growth. Although IGF-I insufficiency is relatively well-tolerated in adulthood, insufficiency can lead to severe consequences in utero through adolescence. ${ }^{59}$ Mutations in the human IGF-I gene that result in abnormal circulating levels of IGF-I are associated with intrauterine and postnatal growth failure, sensorineural deafness, and mental retardation. $^{60,61}$

No controlled studies have been conducted in pregnant or lactating women or in children. However, animal studies suggest that teprotumumab may lead to increased fetal loss, as well as external and skeletal abnormalities. ${ }^{62}$ Therefore, teprotumumab use should be avoided during pregnancy. It is important to screen women for pregnancy prior to initiating treatment and implement effective (such as double barrier) contraception prior to initiation, during, and for 6 months after treatment with teprotumumab. ${ }^{62}$ The authors also strongly recommend that teprotumumab not be used in pediatric patients.

\section{Teprotumumab and Cognitive Changes}

Two patients treated with teprotumumab developed cognitive changes. However, a causal relationship could not be established in either case. The phase 2 trial disclosed 1 case of cognitive changes in a 61-year-old man who developed multiple episodes of transient mental confusion lasting less than 24 hours after 6 doses, without other neurologic symptoms. Brain imaging failed to demonstrate pathologic findings. Lab work revealed elevated thyroid peroxidase antibody. Based on the strong family history of autoimmune thyroid disease and intermittent fluctuating nature of the symptoms, a provisional diagnosis of Hashimoto's encephalopathy was made. Teprotumumab was discontinued even though the association between treatment and these cognitive changes was uncertain. ${ }^{13,46}$

A case report by Hoang et al described a 76-year-old man with Graves' disease, rendered euthyroid with methimazole, who developed 6 weeks of rapidly worsening cognitive decline after the fourth infusion of teprotumumab. The patient developed intermittent behavioral and mental changes including confabulation, memory deficit, and delirium. Teprotumumab was discontinued. Acute neurologic etiologies were ruled out with normal brain 
imaging and laboratory tests. The neurologic condition did not respond to systemic steroids or intravenous immunoglobulins but resolved following plasmapheresis. Causal relationship of this neurologic presentation with teprotumumab was not established. ${ }^{63}$

\section{Conclusion}

Teprotumumab has been shown to be effective in the treatment of active, moderate to severe TED in the phase 2 and phase 3 clinical trials. Subsequent case reports suggest that the drug may also be effective in treating CON and chronic inactive TED. The therapeutic effects of teprotumumab on TED have rapid onset and appear durable. Results from the case reports will need confirmation in larger studies of patients with $\mathrm{CON}$ and inactive TED. There have been no clinical studies comparing teprotumumab with other treatment modalities traditionally used to treat TED (eg medical therapy including corticosteroids, other biologics, and surgical management). Future studies could focus on these comparisons as well as cost-effectiveness analysis of teprotumumab.

Obtaining detailed medical history prior to initiating therapy with teprotumumab is important. This includes details of risk factors for IBD, hearing impairment, hyperglycemia, and ruling-out pregnancy. Teprotumumab is contraindicated in pregnancy. The drug may also exacerbate pre-existing IBD and be associated with hyperglycemia and hearing impairment. Patients with pre-existing IBD should be monitored for disease flare, in which case the drug should be discontinued. Hyperglycemia should be carefully monitored, and diabetes medication should be adjusted as indicated for maintaining optimal glycemic control. Patients should also be closely monitored for the development of hearing impairment. Obtaining routine baseline and periodic pre-infusion audiologic assessment is prudent.

\section{Disclosure}

Dr. Terry J. Smith has been issued the following US patents, held by University of California Los Angeles and Los Angeles Biomedical Institute and covering the use of insulin-like growth factor I receptor inhibition and teprotumumab in Graves' disease and other autoimmune diseases: 6936426, 7998681, 8153121, and 8178304. He receives consulting fees from Horizon Therapeutics and Immunovant outside the submitted work. His institution received research support from Horizon Therapeutics for participating in the clinical trials of teprotumumab for TED. Dr. Shannon S. Joseph receives consulting fees from Horizon Therapeutics outside the submitted work. The authors report no other conflicts of interest in this work.

\section{References}

1. Smith TJ, Laszlo H. Graves' disease. N Engl J Med. 2016;375 (16):1552-1565. doi:10.1056/NEJMra1510030

2. Shan SJC, Douglas RS. The pathophysiology of thyroid eye disease. $J$ Neuroophthalmol. 2014;34(2):177-185. doi:10.1097/WNO.000 0000000000132

3. Tsui S, Naik V, Hoa N, et al. Evidence for an association between TSH and IGF-1 receptors: a tale of two antigens implicated in Graves' disease. J Immunol. 2008;181(6):4397-4405. doi:10.4049/ jimmunol.181.6.4397

4. Ponto KA, Kanitz M, Olivo PD, Pitz S, Pfeiffer N, Kahaly GJ. Clinical relevance of thyroid-stimulating immunoglobulins in Graves' ophthalmopathy. Ophthalmology. 2011;118(11):2279-2285. doi:10.1016/j.ophtha.2011.03.030

5. Lytton SD, Ponto KA, Kanitz M, Matheis N, Kohn LD, Kahaly GJ. A novel thyroid stimulating immunoglobulin bioassay is a functional indicator of activity and severity of Graves' orbitopathy. J Clin Endocrinol Metab. 2010;95(5):2123-2131. doi:10.1210/jc.2009-2470

6. Eckstein AK, Plicht M, Lax H, et al. Thyrotropin receptor autoantibodies are independent risk factors for Graves' ophthalmopathy and help to predict severity and outcome of the disease. J Clin Endocrinol Metab. 2006;91(9):3464-3470. doi:10.1210/jc.2005-2813

7. Eckstein AK, Plicht M, Lax H, et al. Clinical results of antiinflammatory therapy in Graves' ophthalmopathy and association with thyroidal autoantibodies. Clin Endocrinol. 2004;61 (5):612-618. doi:10.1111/j.1365-2265.2004.02143.x

8. Gerding MN, van der Meer JW, Broenink M, Bakker O, Wiersinga WM, Prummel MF. Association of thyrotrophin receptor antibodies with the clinical features of Graves' ophthalmopathy. Clin Endocrinol. 2000;52(3):267-271. doi:10.1046/j.1365-2265.2000.0 0959.x

9. Smith TJ. Insulin-like growth factor pathway and the thyroid. Front Endocrinol (Lausanne). 2021;12:653627. doi:10.3389/fendo.20 21.653627

10. Traisk F, Tallstedt L, Abraham-Nordling M, et al. Thyroid-associated ophthalmopathy after treatment for Graves' hyperthyroidism with antithyroid drugs or iodine-131. J Clin Endocrinol Metab. 2009;94 (10):3700-3707. doi:10.1210/jc.2009-0747

11. Schnitzer T, Kuenkele KP, Rebers F, et al. Characterization of a recombinant, fully human monoclonal antibody directed against the human insulin-like growth factor-1 receptor. Eur $J$ Cancer Suppl. 2006;4(12):66-67. doi:10.1016/S1359-6349(06)70219-1

12. Markham A. Teprotumumab: first approval. Drugs. 2020;80 (4):509-512. doi:10.1007/s40265-020-01287-y

13. Smith TJ, Kahaly GJ, Ezra DG, et al. Teprotumumab for thyroid-associated ophthalmopathy. $N$ Engl $J$ Med. 2017;376 (18):1748-1761. doi:10.1056/NEJMoa1614949

14. Douglas RS, Kahaly GJ, Patel A, et al. Teprotumumab for the treatment of active thyroid eye disease. $N$ Engl J Med. 2020;382 (4):341-352. doi:10.1056/NEJMoa1910434

15. Kahaly GJ, Douglas RS, Holt RJ, Sile S, Smith TJ. Teprotumumab for patients with active thyroid eye disease: a pooled data analysis, subgroup analyses, and off-treatment follow-up results from two randomised, double-masked, placebo-controlled, multicentre trials. Lancet Diabetes Endocrinol. 2021;9(6):360-372. doi:10.1016/ S2213-8587(21)00056-5 Epub 2021 Apr 15.

16. Braun TL, Bhadkamkar MA, Jubbal KT, Weber AC, Marx DP. Orbital decompression for thyroid eye disease. Semin Plast Surg. 2017;31(1):40-45. doi:10.1055/s-0037-1598192 
17. Wu CY, Niziol LM, Musch DC, Kahana A. Thyroid-related orbital decompression surgery: a multivariate analysis of risk factors and outcomes. Ophthalmic Plast Reconstr Surg. 2017;33(3):189-195. doi:10.1097/IOP.0000000000000699

18. Chen J, von Bartheld CS. Role of exogenous and endogenous trophic factors in the regulation of extraocular muscle strength during development. Invest Ophthalmol Vis Sci. 2004;45(10):3538-3545. doi:10.1167/iovs.04-0393

19. Terwee CB, Dekker FW, Mourits MP, et al. Interpretation and validity of changes in scores on the Graves' ophthalmopathy quality of life questionnaire (GO-QOL) after different treatments. Clin Endocrinol. 2001;54(3):391-398. doi:10.1046/j.1365-2265.2001.01241.x

20. United States Food and Drug Administration. Dermatologic and ophthalmic Drugs Advisory Committee meeting sponsor briefing document: teprotumumab for injection. Available from: https:/www.fda. gov/media/133431/download;2019. Accessed September 1, 2021.

21. United States Food and Drug Administration. Dermatologic and Ophthalmic Drugs Advisory Committee meeting briefing document for teprotumumab. Available from: https://www.fda.gov/media/ 133429/download;2019. Accessed September 1, 2021.

22. Bartalena L, Krassas GE, Wiersinga W, et al. For European Group on Graves' Orbitopathy (EUGOGO). Efficacy and safety of three different cumulative doses of intravenous methylprednisolone for moderate to severe and active Graves' orbitopathy. J Clin Endocrinol Metab. 2012;97(12):4454-4463. doi:10.1210/jc.2012-2389

23. van Geest RJ, Sasim IV, Koppeschaar HP, et al. Methylprednisolone pulse therapy for patients with moderately severe Graves' orbitopathy: a prospective, randomized, placebo-controlled study. Eur $J$ Endocrinol. 2008;158(2):229-237. doi:10.1530/EJE-07-0558

24. Zang S, Ponto KA, Kahaly GJ. Clinical review: intravenous glucocorticoids for Graves' orbitopathy: efficacy and morbidity. J Clin Endocrinol Metab. 2011;96(2):320-332. doi:10.1210/jc.2010-1962

25. Gao G, Dai J, Qian Y, Ma F. Meta-analysis of methylprednisolone pulse therapy for Graves' ophthalmopathy. Clin Exp Ophthalmol. 2014;42(8):769-777. doi:10.1111/ceo.12317

26. Stiebel-Kalish H, Robenshtok E, Hasanreisoglu M, Ezrachi D, Shimon I, Leibovici L. Treatment modalities for Graves' ophthalmopathy: systematic review and metaanalysis. J Clin Endocrinol Metab. 2009;94(8):2708-2716. doi:10.1210/jc.2009-0376

27. Mourits MP, van Kempen-harteveld ML, Garcia MB, Koppeschaar HP, Tick L, Terwee CB. Radiotherapy for Graves' orbitopathy: randomised placebo-controlled study. Lancet. 2000;355 (9214):1505-1509. doi:10.1016/S0140-6736(00)02165-6

28. Stan MN, Garrity JA, Carranza Leon BG, Prabin T, Bradley EA, Bahn RS. Randomized controlled trial of rituximab in patients with Graves’ orbitopathy. J Clin Endocrinol Metab. 2015;100(2):432-441. doi:10.1210/jc.2014-2572.

29. Salvi M, Vannucchi G, Curro N, et al. Efficacy of B-cell targeted therapy with rituximab in patients with active moderate to severe Graves' orbitopathy: a randomized controlled study. $J$ Clin Endocrinol Metab. 2015;100(2):422-431. doi:10.1210/jc.2014-3014

30. Perez-Moreiras JV, Gomez-Reino JJ, Maneiro JR, et al. Efficacy of tocilizumab in patients with moderate to severe corticosteroid resistant Graves orbitopathy: a randomized clinical trial. $\mathrm{Am}$ J Ophthalmol. 2018;195:181-190. doi:10.1016/j.ajo.2018.07.038

31. Sears CM, Azad AD, Dosiou C, Kossler AL. Teprotumumab for dysthyroid optic neuropathy: early response to therapy. Ophthalmic Plast Reconstr Surg. 2021;37(Suppl 3):S157-S160. doi:10.1097/ IOP.0000000000001831

32. Slentz DH, Smith TJ, Kim DS, Joseph SS. Teprotumumab for optic neuropathy in thyroid eye disease. JAMA Ophthalmol. 2021;139 (2):244-247. doi:10.1001/jamaophthalmol.2020.5296

33. Hwang CJ, Nichols EE, Chon BH, Perry JD. Bilateral dysthyroid compressive optic neuropathy responsive to teprotumumab. Eur JOphthalmol. 2021;1120672121991042. doi:10.1177/1120672121991042
34. Chiou CA, Reshef ER, Freitag SK. Teprotumumab for the treatment of mild compressive optic neuropathy in thyroid eye disease: a report of two cases. Am J Ophthalmol Case Rep. 2021;22:101075. doi:10.1016/j.ajoc.2021.101075

35. Diniz SB, Cohen LM, Roelofs KA, Rootman DB. Early experience with the clinical use of teprotumumab in a heterogenous thyroid eye disease population. Ophthalmic Plast Reconstr Surg. 2021;37 (6):583-591. doi:10.1097/IOP.0000000000001959

36. Sears CM, Wang Y, Bailey LA, et al. Early efficacy of teprotumumab for the treatment of dysthyroid optic neuropathy: a multicenter study. Am J Ophthalmol Case Rep. 2021;23:101111. doi:10.1016/j. ajoc.2021.101111

37. Ugradar S, Shi L, Wang Y, Mester T, Yang H, Douglas RS. Teprotumumab for non-inflammatory thyroid eye disease (TED): evidence for increased IGF-1R expression. Eye. 2020. doi:10.1038/ s41433-020-01297-w

38. Ozzello DJ, Kikkawa DO, Korn BS. Early experience with teprotumumab for chronic thyroid eye disease. Am J Ophthalmol Case Rep. 2020;19:100744. doi:10.1016/j.ajoc.2020.100744

39. Available from: https://clinicaltrials.gov/ct2/show/NCT04583735. Accessed September 1, 2021.

40. Kurzrock R, Patnaik A, Aisner J, et al. A Phase I study of weekly R1507, a human monoclonal antibody insulin-like growth factor-I receptor antagonist, in patients with advanced solid tumors. Clin Cancer Res. 2010;16(8):2458-2465. doi:10.1158/1078-0432. CCR-09-3220

41. Pappo AS, Patel SR, Crowley JJ, et al. R1507, a monoclonal antibody to the insulin-like growth factor 1 receptor, in patients with recurrent or refractory Ewing sarcoma family of tumors: results of a Phase II Sarcoma Alliance for Research Through Collaboration Study. J Clin Oncol. 2011;29(34):4541-4547. doi:10.1200/JCO.2010.34.0000

42. Pappo AS, Vassal G, Crowley JJ, et al. A phase 2 trial of R1507, a monoclonal antibody to the insulin-like growth factor-1 receptor (IGF-1R), in patients with recurrent or refractory rhabdomyosarcoma, osteosarcoma, synovial sarcoma, and other soft tissue sarcomas. Cancer. 2014;120(16):2448-2456. doi:10.1002/cncr.28728

43. Mahadevan D, Sutton GR, Arteta-Bulos R, et al. Phase 1b study of safety, tolerability and efficacy of R1507, a monoclonal antibody to IGF-1R in combination with multiple standard oncology regimens in patients with advanced solid malignancies. Cancer Chemother Pharmacol. 2014;73(3):467-473. doi:10.1007/s00280-013-2372-x

44. Available from: https://evs.nci.nih.gov/ftp1/CTCAE/CTCAE_4.03/ CTCAE_4.03_2010-06-14_QuickReference_8.5x11.pdf. Accessed September 12, 2021.

45. Vogel WH. Infusion reactions: diagnosis, assessment, and management. Clin J Oncol Nurs. 2010;14(2):E10-21. doi:10.1188/ 10.CJON.E10-E21

46. Schiaffino S, Mammucari C. Regulation of skeletal muscle growth by the IGF1-Akt/PKB pathway: insights from genetic models. Skelet Muscle. 2011;1(1):4. doi:10.1186/2044-5040-1-4

47. Bilbao D, Luciani L, Johannesson B, Piszczek A, Rosenthal N. Insulin-like growth factor-1 stimulates regulatory $\mathrm{T}$ cells and suppresses autoimmune disease. EMBO Mol Med. 2014;6 (11):1423-1435. doi:10.15252/emmm.201303376

48. Ashraf DC, Jankovic I, El-Nachef N, Winn BJ, Kim GE, Kersten RC. New-onset of inflammatory bowel disease in a patient with teprotumumab for thyroid associated ophthalmopathy. Ophthalmic Plast Reconstr Surg. 2021;37(5):e160-e164. doi:10.1097/IOP.000000 0000001943

49. Safo MB, Silkiss RZ. A case of ulcerative colitis associated with teprotumumab treatment for thyroid eye disease. Am J Ophthalmol Case Rep. 2021;22:101069. doi:10.1016/j.ajoc.2021.101069

50. Santos MPC, Gomes C, Torres J. Familial and ethnic risk in inflammatory bowel disease. Ann Gastroenterol. 2018;31(1):14-23. doi:10.20524/aog.2017.0208 
51. Moller FT, Andersen V, Wohlfahrt J, Jess T. Familial risk of inflammatory bowel disease: a population-based cohort study 1977-2011. Am J Gastroenterol. 2015;110(4):564-571. doi:10.1038/ajg.2015.50

52. Kossler A, Sears CM, Dosiou C. Hearing loss and teprotumumab. J Endocrine Soc. 2021;5(Supplement_1):A839-A839. doi:10.1210/ jendso/bvab048.1713

53. Belinsky I, Creighton FX, Mahoney N, et al. Teprotumumab and hearing loss: case series and proposal for audiologic monitoring. Ophthalmic Plast Reconstr Surg. 2021. doi:10.1097/ IOP.0000000000001995

54. Gao L, Nakagawa T. Insulin-like growth factor 1: role in the auditory system and therapeutic potential in otology. Curr Opin Otolaryngol Head Neck Surg. 2020;28(5):286-290. doi:10.1097/MOO.00000 00000000652

55. Iwai K, Nakagawa T, Endo T, et al. Cochlear protection by local insulin-like growth factor-1 application using biodegradable hydrogel. Laryngoscope. 2006;116(4):529-533. doi:10.1097/01. mlg.0000200791.77819.eb

56. Yamamoto N, Nakagawa T, Ito J. Application of insulin-like growth factor-1 in the treatment of inner ear disorders. Front Pharmacol. 2014;5:208. doi:10.3389/fphar.2014.00208

57. Goldman JW, Mendenhall MA, Rettinger SR. Hyperglycemia associated with targeted oncology treatment: mechanisms and management. Oncologist. 2016;21(11):1326-1336. doi:10.1634/ theoncologist.2015-0519
58. Lee HBH, Mariash CN, Yoon MK, et al. Teprotumumab and hyperglycemia guidelines to monitor for hyperglycemia in teprotumumab. Ophthalmic Plast Reconstr Surg. 2021;37(4):393. doi:10.1097/ IOP.0000000000001984

59. Walenkamp MJE, Karperien M, Pereira AM, et al. Homozygous and heterozygous expression of a novel insulin-like growth factor-I mutation. J Clin Endocrinol Metab. 2005;90(5):2855-2864. doi:10.1210/jc.2004-1254

60. Woods KA, Camacho-Hubner C, Savage MO, Clark AJ. Intrauterine growth retardation and postnatal growth failure associated with deletion of the insulin-like growth factor I gene. $N$ Engl J Med. 1996;335 (18):1363-1367. doi:10.1056/NEJM199610313351805

61. Bonapace G, Concolino D, Formicola S, Strisciuglio P. A novel mutation in a patient with insulin-like growth factor 1 (IGF1) deficiency. J Med Genet. 2003;40(12):913-917. doi:10.1136/ jmg.40.12.913

62. Tepezza. Package insert. Horizon Therapeutics. Available from: https://www.hzndocs.com/TEPEZZA-Prescribing-Information.pdf Accessed September 1, 2021.

63. Hoang TD, Nguyen NT, Chou E, Shakir MKM. Rapidly progressive cognitive decline associated with teprotumumab in thyroid eye disease. BMJ Case Rep. 2021;14(5):e242153. doi:10.1136/bcr-2021242153

\section{Publish your work in this journal}

Therapeutics and Clinical Risk Management is an international, peerreviewed journal of clinical therapeutics and risk management, focusing on concise rapid reporting of clinical studies in all therapeutic areas, outcomes, safety, and programs for the effective, safe, and sustained use of medicines. This journal is indexed on PubMed Central, CAS,
EMBase, Scopus and the Elsevier Bibliographic databases. The manuscript management system is completely online and includes a very quick and fair peer-review system, which is all easy to use. Visit http://www.dovepress.com/testimonials.php to read real quotes from published authors. 